\section{Wie qualvoll ist der Alltag auf der Intensivstation?}

\author{
Intensivpatienten leiden möglicherweise mehr als gedacht unter Routine- \\ manövern wie Umlagern oder Absaugen. Das legt eine Pilotstudie aus Schwe- \\ den nahe, in der physiologische Parameter als Marker für Unbehagen detail- \\ liert gemessen und aufgezeichnet wurden.
}

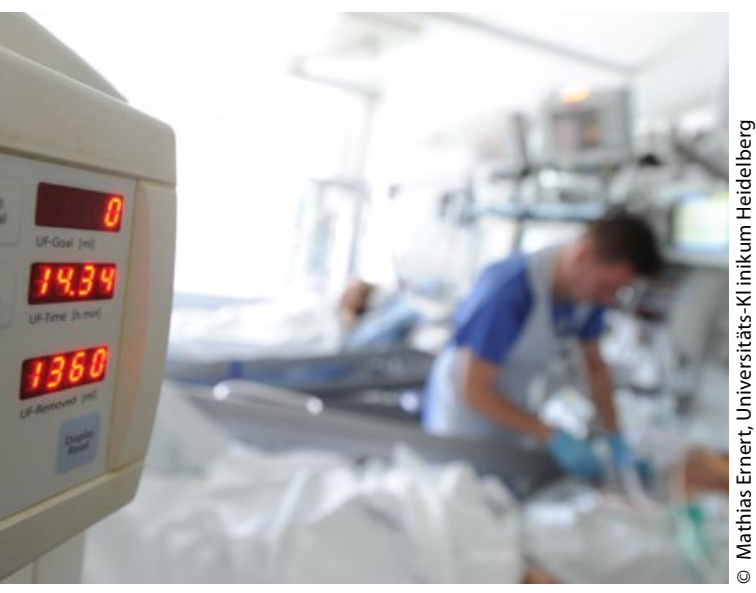

1 ie unangenehm oder gar schmerzhaft viele Routineprozeduren auf der Intensivstation für den schwerkranken, künstlich beatmeten Patienten sein können, lässt sich im Klinikalltag nur erahnen. Intensivpatienten können sich in der Regel nicht mitteilen, und das oft unter Zeitdruck arbeitende Pflegepersonal hat im Routinebetrieb keine Möglichkeit, subtile Reaktionen, die ein Unbehagen anzeigen könnten, zu erfassen und zu dokumentieren.

\section{Studie mit 16 Intensivpatienten}

Diese Mühe hat sich nun ein Forscherteam aus Schweden gemacht: Joakim Engström und Kollegen haben 16 Intensivpatienten - drei Frauen und 13 Männer - über jeweils zwölf Stunden im Tagesbetrieb kontinuierlich überwacht und dabei eine Vielzahl physiologischer Parameter gemessen: von der Atem- und Herzfrequenz über arteriellen Blutdruck und Sauerstoffsättigung bis hin zu Alarmzeichen wie Husten oder Asynchronie zwischen Patient und Respirator. Der Grad der Sedierung wurde mit der Richmond-Agitation-Sedation-Skala (RASS) gemessen.

In die insgesamt 187 Beobachtungsstunden fielen 668 von erfahrenen Intensivpflegekräften ausgeführte Prozeduren. Die Patienten wurden zur Vermei- dung von Druckgeschwüren routinemäßig alle zwei Stunden umgelagert und nach Bedarf abgesaugt, wobei der maximale negative Druck $20 \mathrm{kPa}$ betrug und ein Unterstützungssystem zum Einsatz kam. Das Absaugen erfolgte in 5-Sekunden-Zyklen und durfte für eine Prozedur insgesamt höchstens 20 bis 30 Sekunden dauern.

Während des Beobachtungszeitraums erfasste Engströms Team insgesamt 158 "große“ und 692 „kleinere“ Abweichungen in ihren Messparametern (zur Graduierung hatten die Forscher im Vorfeld für jeden Parameter einen bestimmten Schwellenwert festgelegt). Um mit der Prozedur im Zusammenhang gesehen $\mathrm{zu}$ werden, mussten die Ereignisse innerhalb von 60 Sekunden danach stattgefunden haben.

\section{Abfall der Sauerstoffsättigung, Blutdruckanstieg, Husten}

„Große Abweichungen“ betrafen in erster Linie die Sauerstoffsättigung; der Anteil der Fälle, bei denen der SpO2 unter 90\% gesunken war, lag bei $29 \%$. Am zweithäufigsten war die Asynchronie bei der Beatmung (28\%), gefolgt vom Blutdruckabfall bis zur Hypotension (22\%). Solche großen Ereignisse traten zu 61\% nach Umlagerungsmanövern auf. In $12 \%$ war die Verabreichung eines Medikaments vorausgegangen, in $8 \%$ eine klinische Untersuchung. Wie Engström und Kollegen betonen, waren alle großen Abweichungen in Zusammenhang mit einer Manipulation am Patienten aufgetreten; in ungestörten Ruhephasen wurden keine nennenswerten physiologischen Veränderungen registriert.

Bei den kleineren Ausschlägen standen Blutdruckanstiege oder -abfälle mit $35 \%$ an erster Stelle, gefolgt von Husten (15\%) und einem Anstieg der Atemfrequenz (11\%). Auch hier lag die Ursache in erster Linie bei den Umlagerungsmanövern, gefolgt vom tracheobronchialen Absaugen. Reaktionen zeigten sich aber auch bei der Mundpflege, zu der auch das Absaugen von Speichel unter der Zunge gezählt wurde, oder bei Manipulationen am Endotrachealtubus. Andere Routineprozeduren wie Rasieren, Augenpflege oder Hantieren an der Magensonde schienen die Patienten weniger zu stören; zumindest verursachten sie nur selten kleine Abweichungen in den gemessenen Parametern. Vom Personal dokumentiert wurde keiner der Ausschläge: Pflegende nahmen die Reaktionen der Patienten offenbar als normale Nebeneffekte der Behandlung in Kauf und maßen ihnen dementsprechend keine größere Bedeutung bei.

\section{Umdenken erforderlich!}

Engström und sein Team fordern nun in mehrerer Hinsicht ein Umdenken bei der Pflege von Intensivpatienten. Vor allem die Praxis des Umlagerns sei zu hinterfragen: Es gebe, so die Forscher, keinen wissenschaftlichen Beweis dafür, dass unbedingt ein 2-Stunden-Rhythmus eingehalten werden müsse, um einen Dekubitus zu verhindern. Entsprechende Studien stammen aus einer Ära, in der $\mathrm{u}$. a. die Betten- und Matratzenqualität deutlich schlechter war als heute. Ein wiederholter deutlicher Sättigungsabfall, wie er in der Studie beobachtet wurde, sei bei kritisch kranken Patienten unter Umständen das größere Übel.

In der Studie waren sowohl „Wendemanöver" als auch das tracheobronchiale Absaugen häufig mit einem Blutdruckanstieg verbunden. Bei vielen $\mathrm{Pa}$ tienten lösten die Prozeduren einen Hustenreiz aus, wodurch der Patient aufwachte und sich im Bett bewegte, was oft zu weiteren Problemen führte. Vor diesem Hintergrund sei es unverständlich, so Engström et al., dass so selten Analgetika zum Einsatz kämen.

Die Autoren drängen nun darauf, größere, multizentrische Studien durchzuführen, um Schaden und Nutzen von Routinemaßnahmen auf der Intensivstation besser abwägen zu können und so den Bedürfnissen der schwerkranken Patienten gerecht zu werden.

(eo)

Engström J et al. Physiological changes associated with routine nursing procedures in critically ill are common: an observational pilot study. Acta Anaesthesiol Scand 2016, online 3. November; doi: 10.1111/aas.12827 